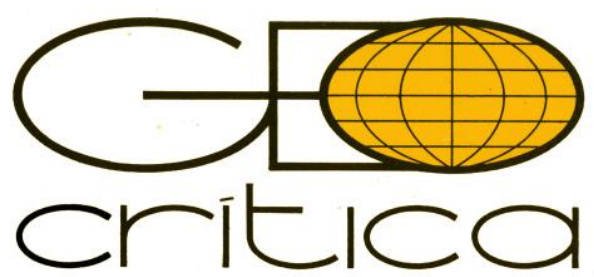

\title{
CONTEXTO GENERAL DE LA AGRICULTURA URBANA EN MÉXICO DURANTE LA COVID-19: USO DE APLICACIONES DE GOOGLE PARA EL ANÁLISIS SOCIAL
}

\author{
Omar Miranda Gómez \\ El Colegio de la Frontera Norte, México \\ omar.mir.go@gmail.com \\ Carlos Rubén Aguilar-Gómez \\ Centro Universitario UAEM Temascaltepec, México \\ craguilarg@uaemex.mx
}

\section{Contexto general de la Agricultura Urbana en México durante la pandemia: uso de aplicaciones de Google para el análisis social (Resumen)}

La Agricultura Urbana (AU) se ha posicionado como una práctica a escala internacional. La crisis sanitaria originada por la COVID-19, generó cambios derivados de la estrategia de confinamiento y distanciamiento social. Uno de ellos, la forma de realizar investigación, mediante nuevas estrategias de recogida de información, como la utilización de herramientas tecnológicas a través de Internet. El presente artículo tiene el objetivo de proponer una ruta metodológica para el análisis de datos mediante el uso de aplicaciones de Google, considerando la práctica de la AU en contexto de la COVID-19. El análisis consistió en recolectar información de grupos de Facebook dedicados a esta actividad en diversos estados de México. Los hallazgos sobre la Agricultura Urbana (AU) en México exponen sus beneficios en distintos aspectos sociales, mientras que el uso de estas herramientas para la investigación social implica ventajas y desventajas.

Palabras clave: Agricultura Urbana, COVID-19, Google forms, Colaboratory, Python. 


\section{General context of Urban Agriculture in Mexico during COVID- 19: use of Google apps for social analysis (Abstract)}

Urban Agriculture (UA) has positioned itself as an international practice. The health crisis caused by COVID-19 generated changes derived from the confinement and social distancing strategy. One of them, how to carry out research, through new information gathering strategies, such as the use of technological tools over the Internet. This article aims to propose a methodological route for data analysis by Google apps, considering the practice of UA in the context of COVID-19. The analysis consisted of collecting information from Facebook groups dedicated to this activity in various states of Mexico. The findings present a general context of this practice and it is concluded that UA assists in many aspects of society, determining some advantages and disadvantages of using these tools for social research.

Key words: Urban Agriculture, COVID-19, Google forms, Colaboratory, Python.

\section{Introducción}

La práctica de la Agricultura Urbana (AU) como se conoce actualmente se originó en las ciudades de Londres, París y Estocolmo, en donde las personas pobres practicaban la AU para tener alimentos disponibles de autoconsumo ${ }^{1}$. En el año 2016, se estimaba que entre el 15 y $20 \%$ de los alimentos a escala global se producen dentro de las ciudades y sus periferias ${ }^{2}$.

La AU puede tener distintas definiciones que contemplan elementos relacionados con las prácticas del campo respecto a la producción de alimentos, ya sea de origen animal o vegetal en zonas urbanas y sus alrededores, teniendo el potencial de producir alimentos para el consumo local e incluso el cultivo de flores y la creación de muros verdes ${ }^{3}$.

Al hablar de Agricultura Urbana, se identifica que ésta comparte características con la agricultura periurbana. No obstante, para el presente artículo, se considera como AU a aquella actividad practicada en pequeñas superficies situadas dentro de una ciudad y destinadas a la producción de cultivos y la cría de ganado menor, para el consumo propio o para la venta en mercados de la vecindad; y es efectuada exclusivamente por personas que viven y trabajan en las ciudades ${ }^{4}$.

La AU contribuye positivamente al medio ambiente en las zonas urbanas, disminuye los gases de efecto invernadero y reduce los desechos urbanos. Además, juega un papel importante en la seguridad alimentaria ${ }^{5}$, la salud, el desarrollo de las economías a escala local, y beneficia

\footnotetext{
${ }^{1}$ Aurora University, 2019 <https://online.aurora.edu/history-of-urban-agriculture/>.

2 Degenhart, $2016<$ https://www.nuso.org/articulo/la-agricultura-urbana-un-fenomeno-global/>.

${ }^{3}$ Kozai et al., 2016 <https://doi.org/10.1016/B978-0-12-801775-3.00003-2>; Orsini et al., 2013 DOI: 10.1007/s13593-013-0143-z.

${ }^{4}$ Zaar, $2011<$ http://www.ub.es/geocrit/b3w-944.htm>.

${ }^{5}$ Aunque la seguridad y soberanía alimentaria pudieran ser conceptualizados como sinónimos, existen posturas que consideran la primera, como la obtención de productos en todo momento sin importar el origen y/o proceso de producción (monocultivo, uso de transgénicos y paquetes tecnológicos), mientras que la segunda se enfoca en la producción local con un enfoque de conservación y respeto ambiental (Gordillo y Méndez, 2013 <http://www.fao.org/3/ax736s/ax736s.pdf>; $\quad$ Zaar, 2015 $<$ https://periodicos.ufrn.br/sociedadeeterritorio/article/view/7870>). Sin embargo, nos ceñiremos a los términos utilizados de los autores citados.
} 
aspectos socio-culturales como la inclusión social, las relaciones de género y el desarrollo de ciencia y tecnología ${ }^{6}$. Por lo tanto, se resalta la multifuncionalidad de la Agricultura Urbana, pues esta actividad adquiere diversos usos como la producción de alimentos, control del microclima, promoción de las áreas verdes, generación de un sentido de comunidad entre los miembros que la practican, conservación de la agrobiodiversidad, como instrumento pedagógico y promotora de la herencia cultural ${ }^{7}$.

Por lo antes mencionado, el aumento en el interés científico por la AU se ha visto reflejado desde la década de 1990, por ejemplo, el Centro Internacional de Investigaciones para el Desarrollo (IDRC por sus siglas en inglés) con sede en Canadá, añadió iniciativas de investigación sobre la AU, y para el año 2000, el Grupo Consultivo sobre Investigación Agrícola (CGIAR por sus siglas en inglés) incluyó esta actividad como una de sus prioridades ${ }^{8}$.

\section{Crisis sanitaria y estrategias de investigación emergentes}

Con la aparición de la COVID-19, se originó una crisis sanitaria mundial, obligando a todos los países a tomar distintas medidas para el cuidado de la población y el control del virus ${ }^{9}$. Algunos efectos de este momento coyuntural se reflejan en el aspecto económico, con impactos negativos, principalmente en la pobreza y la seguridad alimentaria, que se estima, aumentarán en 548 millones y 183 millones respectivamente, y se prevé que la pobreza incremente en 4,4\% en América Latina y el Caribe ${ }^{10}$.

En México, tras el aumento de casos de la COVID-19 el gobierno implementó una estrategia de confinamiento social, donde a partir del 23 de marzo de 2020 se declaró la jornada nacional de sana distancia ${ }^{11}$, cuyo propósito consistió en suspender actividades consideradas no esenciales (recreación y esparcimiento, eventos masivos, actividades educativas, entre otras) con el fin de evitar el contacto entre personas. Esto originó que los individuos se resguardaran y utilizaran su tiempo en distintas labores que les permitieran quedarse dentro de sus residencias. Así, por ejemplo, la ONU en México informó a los ciudadanos cómo realizar un huerto en casa resaltando la AU como una opción ante la emergencia sanitaria, ${ }^{12}$ porque incrementaría la seguridad alimentaria. Sin embargo, para que sea considerada una solución viable derivada ante la COVID-19 deberá de ser reconocida como una ocupación legítima y efectiva ${ }^{13}$.

Ante este contexto, además de los sectores económico, social y político, la academia y la investigación tuvieron que replantearse nuevas estrategias para adentrarse en el análisis de los procesos socio-territoriales. Diversas instituciones comenzaron a problematizar las formas de

\footnotetext{
${ }^{6}$ Josiane y Hugh, 2020 <https://doi.org/10.1007/978-3-319-69626-3_102-1>; Kozai et al., 2016 <https://doi.org/10.1016/B978-0-12-801775-3.00003-2>; Orsini et al., 2013 DOI: 10.1007/s13593-013-0143-z.

${ }^{7}$ Lovell, 2010, p. 2503 <https://www.mdpi.com/2071-1050/2/8/2499>.

${ }^{8}$ Orsini et al., 2013 DOI: 10.1007/s13593-013-0143-z.

${ }^{9}$ OMS, $2020<$ https://www.who.int/es/news/item/29-06-2020-covidtimeline>.

${ }^{10}$ FAO y CEPAL, $2020<$ https://repositorio.cepal.org/bitstream/handle/11362/45722/1/ca9508_es.pdf>.

${ }^{11}$ Gobierno de México.

<https://www.gob.mx/cms/uploads/attachment/file/541687/Jornada_Nacional_de_Sana_Distancia.pdf > .

12 ONU, $2020<$ https://coronavirus.onu.org.mx/agricultura-urbana-una-opcion-ante-la-emergencia-sanitaria>.

${ }^{13}$ Rovelo, 2020 <https://ibero.mx/prensa/huertos-urbanos-solucion-la-vulnerabilidad-alimentaria-por-covid>.
} 
realizar trabajo de campo en tiempos de pandemia ${ }^{14}$. Con base en esta idea, surgió la inquietud de plantear metodologías que ayuden a solventar las actuales limitantes en cuanto a la inmersión de los procesos sociales en tiempos de la COVID-19.

Una alternativa para conocer las opiniones sobre la relevancia de la Agricultura Urbana fue el uso de redes sociales como Facebook y la gestión de datos mediante plataformas digitales. Durante los últimos años, el estudio de esta red social ha cobrado especial interés por los científicos, pues se aprecia como una continua base de datos. Además, se posiciona como una fuente de información del comportamiento humano, difícil de igualar en entornos de investigación más comunes ${ }^{15}$.

En términos de comportamiento humano, hay múltiples fuentes de datos digitales que han comenzado a ser explotadas. En este sentido, las ciencias sociales plantean que la interacción mediada por tecnologías digitales es fundamental porque las personas producen datos en tiempo real (que incluye intermediarios diversos como smartphones, tarjetas de crédito, páginas web, interfaces, detectores o redes sociales, por mencionar algunos), que se van almacenando y pueden ser analizados ${ }^{16}$. Además, la creciente disponibilidad de datos masivos en las redes sociales favorece los esfuerzos por intentar explicar el comportamiento colectivo, ofreciendo oportunidades para mejorar la comprensión de los sistemas en los que viven los seres humanos en la actualidad ${ }^{17}$.

Actualmente, el tratamiento y análisis de datos en el campo de las ciencias sociales pueden ejecutarse utilizando varias herramientas informáticas, de las cuales sobresalen: SPSS, SAS, STATISTICA, Systat, Stata o GenStat ${ }^{18}$, NVIVO, MAXQDA, Atlas.ti. Bajo este contexto, Google ha desarrollado diversas aplicaciones para el análisis y gestión de información, tales como: Docs, Calendar, Spreadsheets, Sites, Drive, My Maps, Colaboratory, Google Translate, Google Scholar, entre otras ${ }^{19}$, las cuales permiten el acceso gratuito mediante la creación de una cuenta Gmail.

En este sentido, por un lado, la Agricultura Urbana se convierte en una actividad que puede paliar la pobreza y aumentar la seguridad y la soberanía alimentaria, además del creciente interés científico por conocer, estudiar y analizar esta actividad; y, por otro lado, la pandemia originada por la COVID-19, generó nuevas estrategias de entrada al campo mediante herramientas tecnológicas para continuar con el estudio de distintos objetos de interés. Por tal razón, el presente artículo tiene el objetivo de proponer una ruta metodológica para el análisis de los procesos sociales mediante aplicaciones de Google, considerando como centro de análisis la práctica de la AU en contexto de la COVID-19 en México. A modo de mantener una estructura argumentativa clara, el artículo se estructuró en tres apartados. El primero, plasma

\footnotetext{
${ }^{14}$ A continuación, se enuncian algunos ejemplos de iniciativas que han abordado la entrada al campo en época de la COVID-19: Dossier: "Pandemia y sociedad. Claves, efectos y desafíos" $<$ http://revistas.ungs.edu.ar/index.php/po/issue/view/3/po25>;

Doing Ethnography Remotely <https://iriss.stanford.edu/doing-ethnography-remotely>; Etnografías de la pandemia por coronavirus <https://revistes.uab.cat/periferia/article/view/v25-n2-fradejas-lubbers-garcia-etal/803-pdf-es>; Etnografía en tiempos de COVID-19 <https://www.iis.unam.mx/blog/etnografia-covid-19/>.

${ }^{15}$ Wilson, Gosling y Graham, 2012, p. 204 <https://journals.sagepub.com/doi/10.1177/1745691612442904>.

${ }^{16}$ González, 2019, p. 269 <https://cintademoebio.uchile.cl/index.php/CDM/article/view/54210>.

${ }^{17}$ Heiberger y Riebling, 2016, p. 1 <DOI: 10.1177/2059799115622763>.

18 Oliden, 2009, p. $652<$ http://www.psicothema.com/pdf/3686.pdf>.

${ }^{19}$ Google, $2021<$ https://about.google/intl/es-419/products/>.
} 
Contexto general de la Agricultura Urbana en México durante la pandemia...

la metodología que siguió la investigación y aborda los materiales y métodos empleados en el análisis; el segundo, presenta los resultados y discusión en torno a la información recabada por los participantes. Y finalmente, se concluye que el uso de estas aplicaciones facilita la recolección de información y posibilita conocer las opiniones de población de diversos contextos territoriales.

\section{Metodología}

El proceso que siguió esta investigación contempló tres fases; la primera, consistió en el diseño de los instrumentos y la determinación de la población objetivo; la segunda, se refiere al trabajo técnico y aplicación del lenguaje de programación; mientras que la tercera, corresponde al análisis de los resultados.

Durante la primera fase se reconoció que el uso de recursos electrónicos por Internet, como los formularios, permiten acercarnos a una realidad espacio-temporal de diversos contextos territoriales con problemas concretos de estudio, además del bajo costo de su implementación y la rapidez en la recolección de $\operatorname{los}$ datos ${ }^{20}$. Al considerar la información anterior, se optó por emplear plataformas colaborativas y de acceso libre asociadas entre sí, en este sentido, las aplicaciones que brinda Google, permiten integrar diversas herramientas: Google Forms, Spreadsheets $^{21}$ y Colaboratory.

Aunque existen diferentes alternativas de formularios como Surveymonkey, Polldaddy, encuestas de Excel en línea, y los formularios de Google, se seleccionó esta última opción para la recolección y análisis de datos, ya que esta alternativa, es flexible, se puede personalizar y es gratuita ${ }^{22}$. Además, permite la vinculación directa de la entrada con hojas de cálculo a través de la pestaña "Respuestas"23.

Respecto al lenguaje de programación encontramos opciones como: $R$, SAS y Python. Para este caso se optó por emplear Python porque utiliza un lenguaje generalista que permite hacer programación orientada a objetos, y también es uno de los lenguajes de programación más utilizados para el análisis de datos ${ }^{24}$, porque ofrece algunas ventajas como el enfoque en la legibilidad y accesibilidad del código, que es especialmente importante para los científicos sociales, quienes en general han recibido poca o ninguna formación en programación y codificación ${ }^{25}$. En este caso, Google cuenta con el notebook Colaboratory el cual permite trabajar con Python y visualizar la información programada sin la necesidad de instalar el software en un ordenador.

Una vez seleccionada la herramienta a utilizar para la recolección de datos (Google Forms), se buscó conocer el panorama general de la Agricultura Urbana en México y su relevancia ante el contexto de la COVID-19. Dicho formulario contempló 15 preguntas relacionadas con la

\footnotetext{
${ }^{20}$ Díaz de Rada, 2012 <https://www.raco.cat/index.php/Papers/article/view/248512〉.

${ }^{21}$ Aplicación de Google drive que permite trabajar hojas de cálculo en la nube sin la necesidad de instalar el software.

${ }^{22}$ Abundis, 2016, p. 178 <https://dialnet.unirioja.es/servlet/articulo?codigo=7286080>.

${ }^{23}$ Wiemken et al., 2018, p. 2 <https://www.sciencedirect.com/science/article/abs/pii/S0196655318300312>.

24 Talens, 2020, p. $21<$ https://riunet.upv.es/handle/10251/156675>.

${ }^{25}$ Heiberger y Riebling, 2016, p. 2. DOI: 10.1177/2059799115622763.
} 
ubicación de los participantes, edad, sexo, tamaño del huerto, diversidad de cultivos que han sembrado, fuentes de abastecimiento de semillas, fuentes y modos de aprendizaje de la AU, tiempo destinado a la actividad, motivaciones y ventajas de la Agricultura Urbana, las emociones en la AU e importancia de la agricultura en el contexto de la COVID- $19^{26}$.

Para la recolección de información, se publicó una encuesta en diversos grupos de Facebook dedicados a la promoción de la Agricultura Urbana en México, esto con el propósito de tener una población objetivo representativa. La búsqueda de estos grupos consideró una escala espacial, pues procuró incorporar al menos un grupo de cada entidad federativa con la intención de obtener una visión nacional de la AU. No obstante, la recolección de información llegó al punto de saturación donde no pudo abarcar los 31 estados de la República Mexicana. Por lo tanto, el estudio solo sondeó 32 grupos repartidos en 25 estados, de los cuales se obtuvieron 87 respuestas (cuadro 1). El formulario estuvo activo durante 19 días (del 17 de septiembre, al 05 de octubre de 2020) y registró un promedio de respuesta de 4,5 participaciones por día. Cabe resaltar que, al inicio de la aplicación del formulario, se registró la mayor incidencia de participaciones.

Cuadro 1. Participación y grupos de Facebook dedicados a la Agricultura Urbana en México

\begin{tabular}{|c|c|c|}
\hline Estado & Grupos de Facebook* & Participación \\
\hline Baja California & Huerto urbano tijuana & 9 \\
\hline \multirow{2}{*}{ Baja California Sur } & Huerto Urbano Los Cabos(\#jardinkrishna) & \multirow[t]{2}{*}{1} \\
\hline & Huertos urbanos La Paz, BCS & \\
\hline \multirow{2}{*}{ Chihuahua } & Huertos Urbanos Juárez El Paso & \multirow[t]{2}{*}{8} \\
\hline & Huertos urbanos Chihuahua, Proyecto & \\
\hline Chiapas & Red Huertos Chiapas & 0 \\
\hline Durango & Huertos urbanos Durango & 0 \\
\hline \multirow[t]{2}{*}{ Estado de México } & Huertos Urbanos Toluca & 4 \\
\hline & Huertos Urbanos en México, DF y Edo. de Méx. & \\
\hline \multirow{3}{*}{ Ciudad de México } & Huertos urbanos CDMX & \multirow[t]{3}{*}{5} \\
\hline & Huerto urbano de la ciudad de México & \\
\hline & Huertos Urbanos México & \\
\hline \multirow{2}{*}{ Guanajuato } & Red de Agricultura Urbana de León & \multirow[t]{2}{*}{8} \\
\hline & El Huerto Urbano Guanajuato & \\
\hline Guerrero & ---- & 1 \\
\hline Jalisco & Huertos urbanos Guadalajara & 5 \\
\hline Michoacán & Huertos urbanos Michoacán -Red- & 2 \\
\hline Morelos & Huertos Urbanos Cuernavaca & 0 \\
\hline Nuevo León & Huertos orgánicos en monterrey & 0 \\
\hline Oaxaca & Huertos y Semillas Oaxaca & 2 \\
\hline
\end{tabular}

${ }^{26}$ Link del formulario <https://forms.gle/auM6ouYVNA3CDGh27>. 
Contexto general de la Agricultura Urbana en México durante la pandemia...

\begin{tabular}{|c|c|c|}
\hline & Mi huerto orgánico - Oaxaca & \\
\hline & Huertos Urbanos Oaxaca & \\
\hline Puebla & ---- & 3 \\
\hline \multirow{2}{*}{ Querétaro } & Principiantes en huertos Querétaro & \multirow[t]{2}{*}{2} \\
\hline & Huertos Urbanos en Querétaro & \\
\hline Quintana Roo & Huertos urbanos Playa del Carmen & 0 \\
\hline San Luis Potosí & Huertos Urbanos SLP & 2 \\
\hline Sinaloa & ---- & 1 \\
\hline Sonora & Huertos caseros en Hermosillo & 0 \\
\hline \multirow{2}{*}{ Tabasco } & Agricultura urbana Tabasco & \multirow[t]{2}{*}{1} \\
\hline & Huertos Tabasco & \\
\hline Veracruz & Red de Agricultura Urbana y Periurbana de Xalapa & 17 \\
\hline Tamaulipas & Huertos Urbanos Tampico & 0 \\
\hline Yucatán & $\begin{array}{l}\text { Huertos Urbanos-Merida,Yuc Mexico: Intercambio de semillas } \\
\text { ORGANICAS }\end{array}$ & 13 \\
\hline Zacatecas & Agricultores urbanos de Zacatecas & 3 \\
\hline & Total de participantes & 87 \\
\hline
\end{tabular}

Fuente: Elaboración propia con base en Facebook <https://www.facebook.com/>.

*Se respetó el nombre de los grupos de Facebook como se presentaron en la búsqueda.

Asimismo, al generar una división regional se pudo apreciar la relevancia de la AU en los diversos estados, pues se reconoció un mayor interés en lugares como Veracruz, Yucatán y Baja California (cuadro 1).

En cuanto a la fase dos, referente al trabajo técnico y procesamiento de datos. Se consideró agrupar y sistematizar la información de las respuestas obtenidas en Google Forms, posteriormente se exportó a una hoja de cálculo de Spreadsheets, y finalmente se asoció con la aplicación Colaboratory, con la intención de analizar las opiniones vertidas en el formulario. Se realizó un procesamiento manual de la información con el propósito de agrupar las fuentes y modos de aprendizaje, edades de los participantes, motivaciones, y ventajas sobre la práctica de la AU. Esta aplicación, al estar asociada con las hojas de cálculo de la plataforma Drive, permite procesar la información sin necesidad de almacenarla en dispositivos electrónicos y es de uso gratuito. Además, es una buena opción para el modelado, ya que ahorra costos de computación en lo que respecta a la ciencia de datos y la creación de prototipos de aprendizaje automático ${ }^{27}$.

El proceso de análisis mediante Colaboratory consideró los siguientes pasos: 1) importación de bibliotecas, 2) importación de base de datos, 3) análisis de texto, y 4) visualización gráfica. Para analizar el contenido de las preguntas abiertas, se importaron las bibliotecas pandas, $n l t k$ y matplotlib. Pandas, permite una implementación eficiente de un DataFrame (matrices multidimensionales con etiquetas de fila y columna adjuntas que a menudo cuentan con información heterogénea y/o datos faltantes), ofrece una interfaz de almacenamiento

${ }^{27}$ Bisong 2019, p. 64. 
conveniente para datos etiquetados, y ejecuta una serie de operaciones para usuarios familiarizados con las bases de datos y programas de hojas de cálculo ${ }^{28}$.

Por su parte, el análisis de texto se refiere al proceso de representación y modelado de contenido textual que ayuda a descubrir patrones en el discurso ${ }^{29}$. Para efectuar el análisis, Python cuenta con la biblioteca NLTK (natural language toolkit) que permite analizar el "lenguaje natural", entendido como el lenguaje que usamos cada día para comunicarnos. En este caso, se empleó un "procesamiento del lenguaje natural" que consiste en la manipulación informativa del lenguaje y ayuda a comprender las expresiones humanas con el fin de darles una explicación ${ }^{30}$.

Una vez teniendo en cuenta los requerimientos del software, el análisis del texto consideró tres momentos: 1) recolección de información de tipo textual, 2) eliminación de stopwords, que corresponde al filtrado de palabras o frases que no transmiten información significativa sobre el texto ${ }^{31}$, y 3) aglomeración por palabras.

Finalmente, en cuanto a la visualización de la información, se optó por el uso de word clouds ya que proporciona una representación gráfica del conocimiento, que permite al espectador formar un sentido rápido e intuitivo de un texto. Esta herramienta es una forma sencilla de compartir datos de alto nivel sin sobrecargar la información para el usuario ${ }^{32}$. En este caso, para desarrollar la nube de palabras se empleó la biblioteca matplotlib, porque permite el trazado de gráficos 2D y 3D que utiliza el lenguaje de programación Python, con calidad de publicación científica en una variedad de formatos impresos y entornos interactivos ${ }^{33}$.

\section{Resultados y discusión}

Los resultados obtenidos se presentan de acuerdo con las fases establecidas en la metodología, retomando 87 formularios provenientes de individuos que practican la AU. De los cuales el 77,3\% fueron mujeres, $19,3 \%$ fueron hombres y el 3,4\% prefirió no decirlo.

\section{Fuentes y modos de aprendizaje de la Agricultura Urbana}

De los resultados obtenidos, se concluye que la principal fuente de aprendizaje es Internet (figura 1). En este sentido se reporta que en México para el periodo 2015-2018, el uso de plataformas con contenidos audiovisuales gratuitos aumentó el $19,7 \%{ }^{34}$. Aunque no se puede asegurar que todos los individuos han visualizado contenidos de información sobre AU, esta cifra podría ayudar a relacionar la fuente de aprendizaje, además de las nuevas tendencias en

\footnotetext{
28 VanderPlas, 2016, p. 97.

${ }^{29}$ Srinivasa, Siddesh y Srinidhi 2018, p. 219.

30 Bird, Klein y Loper, 2009, p. ix.

${ }^{31}$ Srinivasa, Siddesh y Srinidhi, 2018, p. 220.

32 Depaolo y Wilkinson, 2014, p. 44 <https://link.springer.com/article/10.1007/s11528-014-0750-9>.

${ }^{33}$ Ari y Ustazhanov, 2014, p. 1 <https://ieeexplore.ieee.org/document/6997585>.

34 IFT, 2019 <http://www.ift.org.mx/estadisticas/uso-de-las-tic-y-actividades-por-internet-en-mexico-impactode-las-caracteristicas-sociodemograficas-0>.
} 
la alimentación (thinspiration, fitspiration, movimiento slowfood) ${ }^{35}$. A su vez, se identificó que los sujetos han aprendido sobre la Agricultura Urbana a través de cursos y manuales. A escala internacional, se ha sugerido la promoción de la educación ambiental para contribuir a la seguridad alimentaria, mediante cursos de AU, que diferentes instituciones educativas, organizaciones no gubernamentales, y asociaciones civiles están ofertando lo que expresa una oportunidad más para conocer y practicar dicha actividad ${ }^{36}$.

Figura 1. Fuentes y modos de aprendizaje de la AU

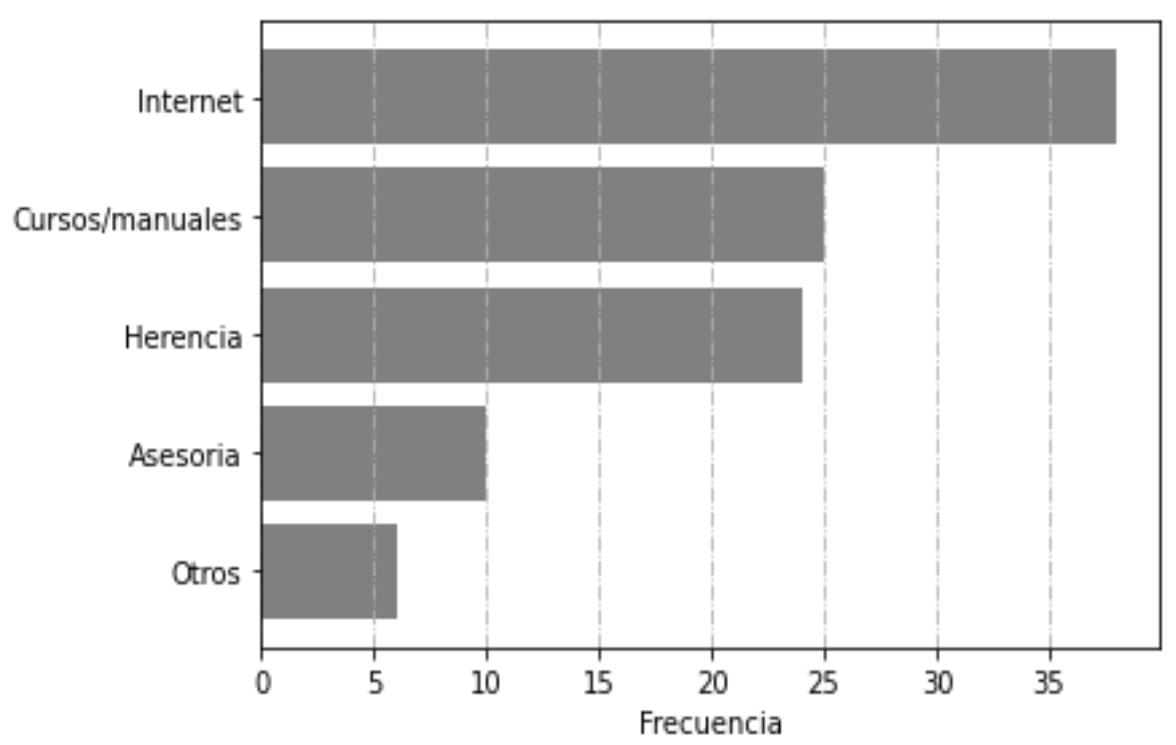

Fuente: Elaboración propia mediante las respuestas obtenidas en Google Forms.

Otro componente que se posiciona como un elemento que influye en la práctica de la Agricultura Urbana, es la herencia o tradición familiar. Este podría ser el único que no tiene un origen o interés económico, ambiental o de salud, sino que se encuentra relacionado a un aspecto cultural, pues se ha visto reflejado en diferentes estudios que no solo dan muestra de su importancia social como parte de las costumbres y tradiciones, sino que mantienen los conocimientos a través del tiempo ${ }^{37}$. A pesar de que existen nuevas tecnologías para la práctica de la AU, se ha identificado una preferencia por las técnicas tradicionales para la producción ${ }^{38}$, como el uso de semillas nativas, abono orgánico y el policultivo.

\footnotetext{
35 Marcos, 2019

$<$ https://repositorio.uam.es/bitstream/handle/10486/687899/marcos_retuerta_saratfg.pdf?sequence=1\&isAllowe $\mathrm{d}=\mathrm{y}>$.

${ }^{36}$ Chávez y Palacios, $2017<$ https://dialnet.unirioja.es/servlet/articulo?codigo=6500073>.

${ }^{37}$ McClintock, 2010 <DOI:10.1093/cjres/rsq005>; Vásquez, $2010<\mathrm{DOI}: 10.1093 /$ cjres/rsq005>.

${ }^{38}$ Philpott et al., $2020<$ https://doi.org/10.5751/ES-11666-250408>; Peña y Veselovschi, 2019

$<$ https://bdigital.uexternado.edu.co/bitstream/001/2247/1/DQA-spa-2019-

La_agricultura_urbana_como_herramienta_para_el_bienestar_de_las_personas_mayores>; Nadal et al., 2018.

<https://www.sciencedirect.com/science/article/abs/pii/S0264837717301096>; Méndez et al., 2005

$<$ https://www.redalyc.org/articulo.oa?id=11705504>.
} 
De acuerdo con el Instituto Federal de Telecomunicaciones (IFT) (2019), la población que tiene más probabilidad de utilizar Internet se sitúa entre 12 y 44 años de edad. Dichos datos concuerdan con los resultados obtenidos en la presente investigación, ya que los individuos que contestaron a las respuestas poseen entre 25 y 44 años (figura 2). Sin embargo, no solo la edad influye, sino que otras características como el nivel educativo y el nivel de ingresos económicos, lo cual es un elemento importante ya que se evidencia que esta práctica puede no ser accesible para todos y solo para aquellas personas con solvencia económica.

Figura 2. Edades de los participantes

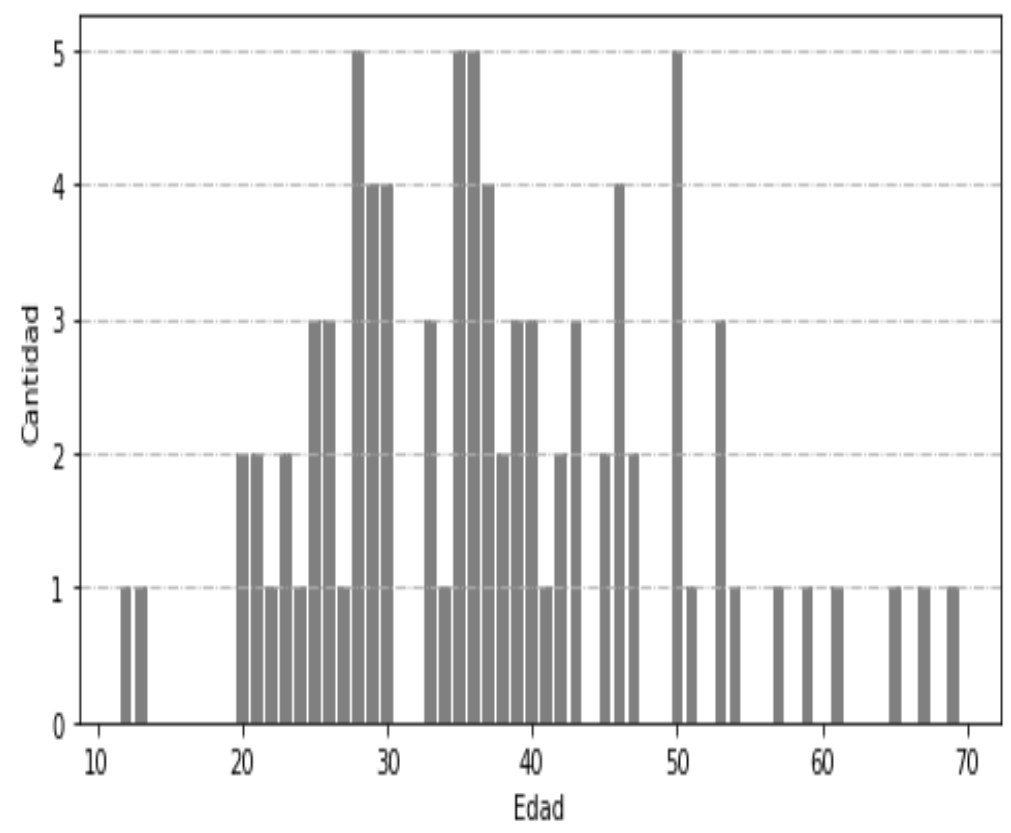

Fuente: Elaboración propia mediante las respuestas obtenidas en Google Forms.

\section{Motivaciones y ventajas de la Agricultura Urbana}

La FAO menciona que la AU busca la seguridad alimentaria mediante la producción de alimentos para el autoconsumo en espacios reducidos ${ }^{39}$. Al menos tres elementos de este argumento se identifican en los hallazgos de la presente investigación: seguridad alimentaria, autoconsumo y espacio. En este caso, los resultados muestran que las principales motivaciones para la práctica de la agricultura en México se relacionan al autoconsumo y la salud (figura 3), que está asociado con la soberanía alimentaria ${ }^{40}$, lo cual está ligado a la calidad del suelo donde se cultivan y el agua con que son regados ${ }^{41}$, asegurando la inocuidad de los alimentos. Aunque no se estipula una pregunta relacionada al agua utilizada en la práctica de la Agricultura Urbana

\footnotetext{
${ }^{39}$ FAO, $2012<$ http://www.fao.org/3/as174s/as174s.pdf>.

40 Zaar, $2015<$ https://periodicos.ufrn.br/sociedadeeterritorio/article/view/7870>.

${ }^{41}$ Philpott et al., 2020 <https://doi.org/10.5751/ES-11666-250408>; Soriano, 2005 citado en Vásquez Moreno, 2010 <https://www.colef.mx/posgrado/wp-content/uploads/2011/04/TESIS-V\%C3\%A1squez-Moreno-LarissaMAIA.pdf $>$.
} 
se parte de que el líquido proviene de fuentes como la red de agua potable y el agua de lluvia. Estas motivaciones se han reportado en otros estudios ${ }^{42}$. Respecto al espacio, el $36,4 \%$ de los individuos que practican Agricultura Urbana tienen un tamaño promedio de huerto de tres a cinco metros cuadrados seguidos del $22,7 \%$ que presentan entre uno y dos metros cuadrados y solo el $15,9 \%$ entre seis y ocho metros, lo cual indica que, desde un punto de vista productivo, la práctica de la AU se está desarrollando en espacios reducidos. No obstante, hay que considerar el tipo de productos que se obtienen en dichos espacios ya que el diseño, el sustrato, disponibilidad de agua y la profundidad son variables que influyen en el éxito o fracaso del sistema agrícola ${ }^{43}$.

Figura 3. Motivaciones

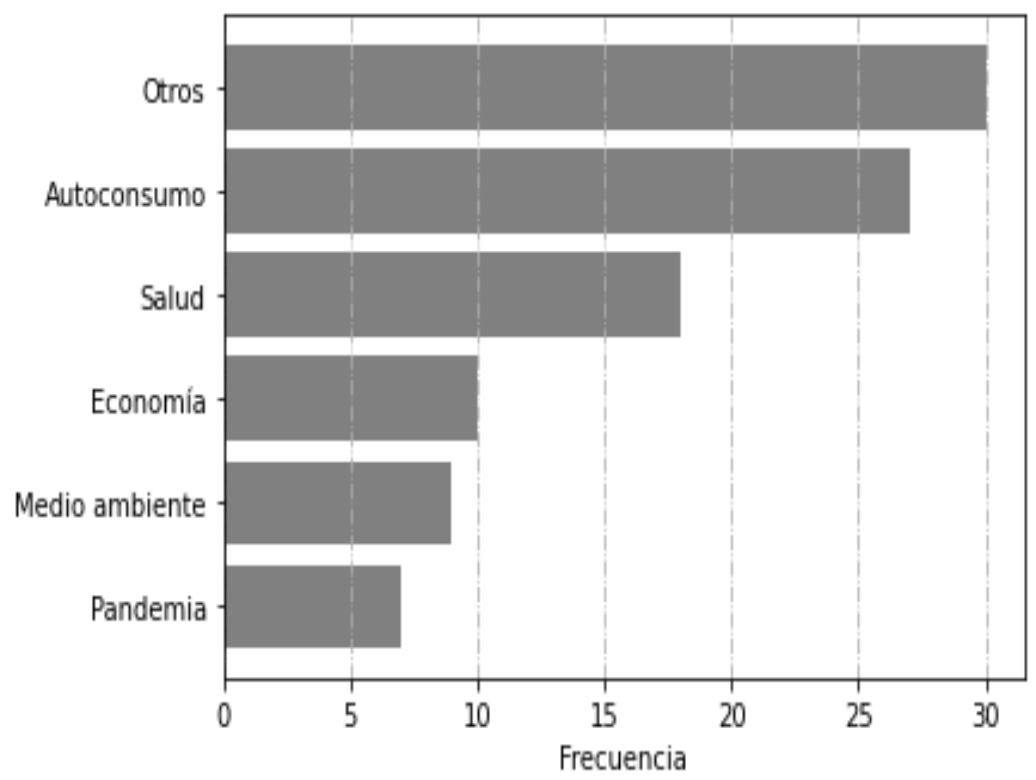

Fuente: Elaboración propia mediante las respuestas obtenidas en Google Forms.

El factor económico es la tercera actividad que motiva la práctica de la Agricultura Urbana, lo cual podría relacionarse con la resiliencia económica y sustentabilidad urbana ${ }^{44}$; en este sentido, diferentes investigaciones sobre la práctica de la AU mencionan como primera motivación el factor económico relacionado a la necesidad, precariedad y falta de trabajo ${ }^{45}$. En algunos países como México este factor se desenvuelve en un contexto que incluye falta de oportunidades laborales y pagos deficientes, lo cual refleja este tipo de estrategias para la reducción de gastos. Por ejemplo, las respuestas obtenidas refieren que el factor económico es un elemento importante para la práctica de la $\mathrm{AU}$, sin embargo, las menciones a esta respuesta se asocian con el autoconsumo y el ahorro para no generar gastos, es decir, no se dedican a la

\footnotetext{
42 Vásquez Moreno, 2010 <https://www.colef.mx/posgrado/wp-content/uploads/2011/04/TESISV\%C3\%A1 squez-Moreno-Larissa-MAIA.pdf $>$.

${ }^{43}$ Agromática, 2012 <https://www.agromatica.es/jardin/>; Portalfruticola, 2018

$<$ https://www.portalfruticola.com/noticias/2018/11/12/guia-para-principiantes-para-el-cultivo-de-hortalizas-yfrutas-en-espacios-reducidos/>.

${ }^{44}$ McClintock, 2009 DOI:10.1093/cjres/rsq005.

45 Degenhart, 2016 <https://www.nuso.org/articulo/la-agricultura-urbana-un-fenomeno-global/>; Méndez et al., $2005<$ https://www.redalyc.org/articulo.oa?id=11705504>.
} 
comercialización del producto. El interés por el medio ambiente también ocupa un lugar importante dentro de las motivaciones por parte de los sujetos y en este sentido algunos estudios también han identificado esta tendencia ${ }^{46}$. En los últimos 40 años, con la aparición del desarrollo sustentable, se han llevado campañas de concientización y conservación del medio ambiente, que podrían estar influyendo en esta motivación.

Aunque la investigación se realizó en 2020, considerando la pandemia originada por la COVID-19, las respuestas obtenidas en cuanto a la motivación muestran que este problema de salubridad mundial no ha influido en el interés por la práctica de la AU. Distintos elementos podrían responder a esto; por ejemplo, el tiempo que los sujetos llevan practicando la agricultura, quienes, además de las motivaciones antes expuestas, mencionaron la herencia de la práctica por parte de sus antepasados, el espacio, la recreación, curiosidad, experimentación, estudios relacionados y espiritualidad. De estos elementos, se reporta que la expresión de antecedentes rurales o tradición familiar, además de la recreación, son elementos motivadores para la práctica de la $\mathrm{AU}^{47}$. Esto quiere decir que la práctica de la $\mathrm{AU}$ es anterior a la pandemia COVID-19. Otro aspecto puede relacionarse, a los contextos en donde se desenvuelven los sujetos, quienes presentan condiciones económicas limitadas además de la falta de seguridad y soberanía alimentaria, que los llevan a buscar estrategias para disminuir estas problemáticas.

Figura 4. Ventajas sobre la práctica de la AU

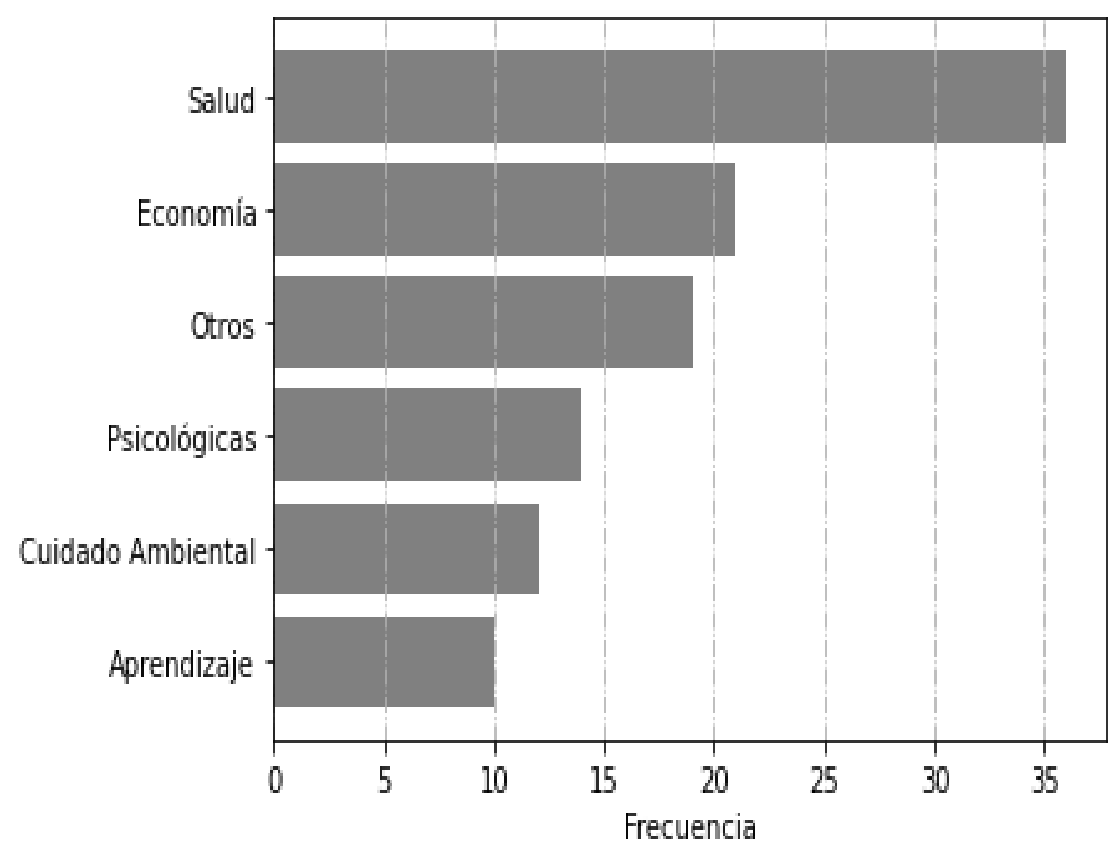

Fuente: Elaboración propia mediante las respuestas obtenidas en Google Forms.

\footnotetext{
${ }^{46}$ Degenhart, 2016 <https://www.nuso.org/articulo/la-agricultura-urbana-un-fenomeno-global/>; Soriano, 2005 citado en Vásquez Moreno, $2010<$ https://www.colef.mx/posgrado/wp-content/uploads/2011/04/TESISV\%C3\%A1 squez-Moreno-Larissa-MAIA.pdf $>$.

${ }^{47}$ Soriano, 2005 citado en Vásquez Moreno, $2010<$ https://www.colef.mx/posgrado/wpcontent/uploads/2011/04/TESIS-V\%C3\%A1squez-Moreno-Larissa-MAIA.pdf>; Méndez et al., 2005 <https://www.redalyc.org/articulo.oa?id=11705504>.
} 
Se identifica una relación importante entre las ventajas y las motivaciones antes mencionadas, en este caso, la salud y la economía aparecen comprometidas en ambas imágenes (figura 3 y figura 4). Para el caso de la salud, los sujetos consideran que se encuentra relacionada al "control" de dichos alimentos, en dónde quién los produce los consume, y por lo tanto son saludables, además, diferentes estudios concuerdan en que los sujetos perciben esta práctica como "sana" debido a que no utiliza pesticidas ${ }^{48}$. Sin embargo, es importante mencionar que los sujetos no conocen realmente el origen de las semillas que adquirieron y algunos de ellos las compraron en supermercados y aunque esta es la principal ventaja, no existe certeza de que dichos alimentos sean saludables ya que se necesita conocer la cantidad de nutrientes que contienen (proteínas, carbohidratos, grasas, vitaminas y minerales), la forma de consumirlos y el tipo de alimentación, y si las prácticas no son bien ejecutadas podrían tener efectos negativos en la salud. Por ejemplo, conocer la composición, estructura y nutrientes del suelo (principalmente nitrógeno, fósforo y potasio) permite que los productos cultivados adquieran los elementos necesarios para su desarrollo ${ }^{49}$.

Por otra parte, la economía es también un factor preponderante en dichas ventajas identificadas, influyendo en las principales motivaciones para la práctica de Agricultura Urbana, porque reduce el gasto para la obtención de alimentos, aunque se necesita realizar un análisis más profundo para conocer el costo-beneficio de la práctica de la AU. Un estudio en Mérida (México), menciona que los residentes consideran que sus ingresos no son suficientes para desarrollar y mantener los costos de sus "sistemas agrícolas"50. En tal caso se podría pensar que la AU puede ser practicada por personas con ingresos económicos suficientes, a pesar de que esta práctica busca la seguridad alimentaria para las poblaciones vulnerables urbanas y periurbanas ${ }^{51}$. Desde este planteamiento, podría existir un problema de equidad de la AU en donde "quien puede lo practica". En este sentido, se ha propuesto que la agricultura y la salud son sectores que deben colaborar mediante el liderazgo de instituciones que logren políticas y una buena gobernanza que facilite la integración, con capacidad a todos los niveles para generar un verdadero impacto $^{52}$. Es decir, buscar desde el gobierno y otras esferas como las instituciones gubernamentales y ONG's, la promoción y generación de planes y programas que inciten a la población para llevar a cabo esta práctica.

\section{Las emociones en la Agricultura Urbana}

Las características psicológicas identificadas podrían resaltar aún más la importancia de dicha actividad, lo que sugiere, que la AU no es solo una práctica que mejorará las condiciones fisiológicas del sujeto, sino que a nivel psicológico estará influyendo positivamente en la misma $^{53}$. En este sentido, se menciona que los principales elementos se relacionan a los

\footnotetext{
${ }^{48}$ Nadal et al., 2018 <https://www.sciencedirect.com/science/article/abs/pii/S0264837717301096>; McClintock, 2010. DOI:10.1093/cjres/rsq005.

${ }^{49}$ FAO, $2000<$ http://www.fao.org/3/V5290S/v5290s28.htm>.

${ }^{50} \mathrm{Nadal}$ et al., 2018 <https://www.sciencedirect.com/science/article/abs/pii/S0264837717301096>.

${ }^{51}$ FAO, $2012<$ http://www.fao.org/3/as174s/as174s.pdf $>$.

52 Hawkes y Ruel, 2006 <https://www.scielosp.org/pdf/bwho/2006.v84n12/984-990/en>.

${ }^{53}$ McClintock, 2010 DOI:10.1093/cjres/rsq005.
} 
recuerdos en el campo y las emociones, que otorgan tranquilidad, seguridad alimentaria y autonomía $^{54}$. Dichos recuerdos podrían estar relacionados a la herencia familiar y tradición.

La nube de palabras (figura 5) muestra que los sujetos identificaron una relación entre la AU y diferentes sentimientos y emociones como, amor, relajación, pasión, felicidad, satisfacción. De estas, se identificó que la relajación y la satisfacción se han reportado en otros estudios ${ }^{55}$, relacionados a esta práctica. Además de que la influencia de la Agricultura Urbana es benéfica, pues se utiliza como terapia, de manera positiva, ocupa el tiempo, se producen alimentos, ayuda con el estrés, genera paz, tranquilidad y felicidad (figura 6). En este sentido, diferentes estudios explican que la AU puede influir psicológicamente en los individuos, por ejemplo, el manejo del estrés ${ }^{56}$ e incluso, se ha identificado que el ser humano, al estar en contacto con las prácticas ambientales, recupera el desgaste psicológico que se genera para satisfacer los requerimientos de la vida diaria, además de que se reconoce que genera salud mental ${ }^{57}$.

Figura 5. Relación de la AU con los sentimientos y las emociones

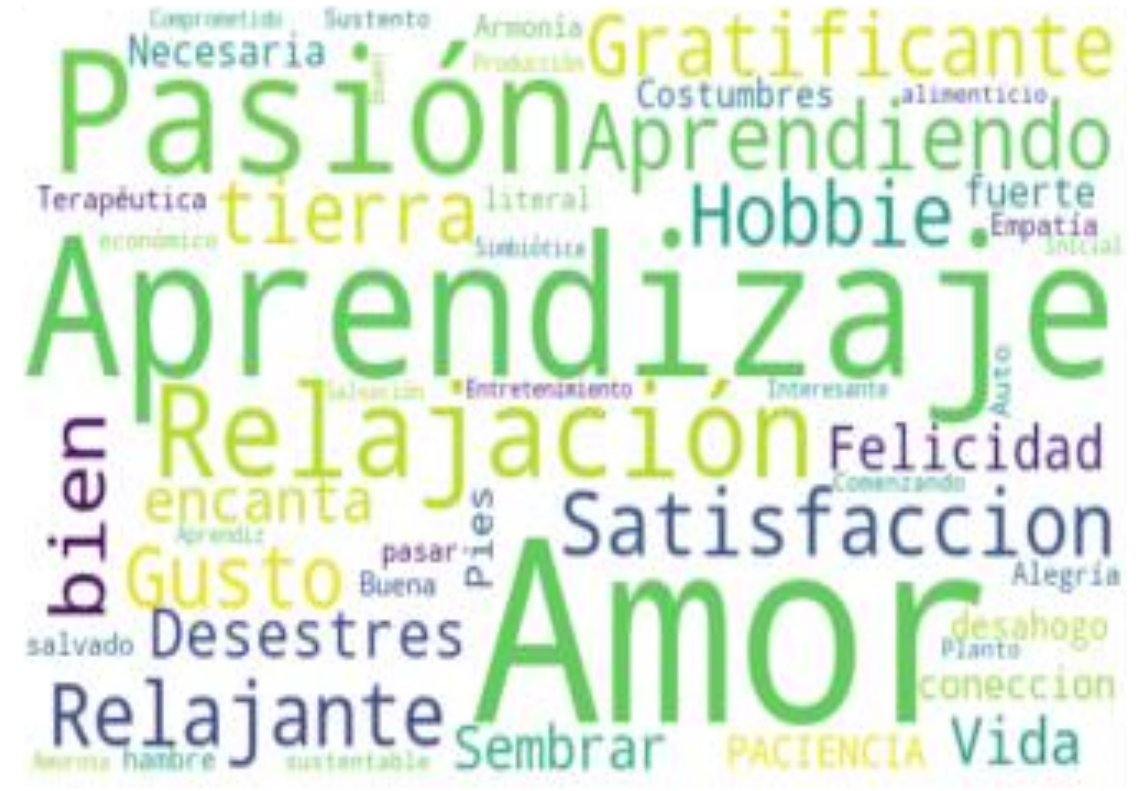

Fuente: Elaboración propia mediante el uso de Colaboratory.

\footnotetext{
54 Cantor, $2010<$ http://www.scielo.org.co/scielo.php?script=sci_arttext\&pid=S0122$14502010000200004 \& \operatorname{lng}=$ es\&nrm=iso $>$.

${ }^{55}$ Nadal et al., 2018 <https://www.sciencedirect.com/science/article/abs/pii/S0264837717301096>; Vásquez Moreno $2010<$ https://www.colef.mx/posgrado/wp-content/uploads/2011/04/TESIS-V\%C3\%A1squez-MorenoLarissa-MAIA.pdf $>$.

${ }^{56}$ Mejías, 2014 <https://institucional.us.es/revistas/habitat/6/HyS\%20n06a04.pdf>.

57 Grajales y Peñaloza, 2019

<https://repository.ucc.edu.co/bitstream/20.500.12494/13421/1/2019_aspectos_salud_mental.pdf>; Peña y

Veselovschi, 2019 <https://bdigital.uexternado.edu.co/bitstream/001/2247/1/DQA-spa-2019-

La_agricultura_urbana_como_herramienta_para_el_bienestar_de_las_personas_mayores $>$.
} 


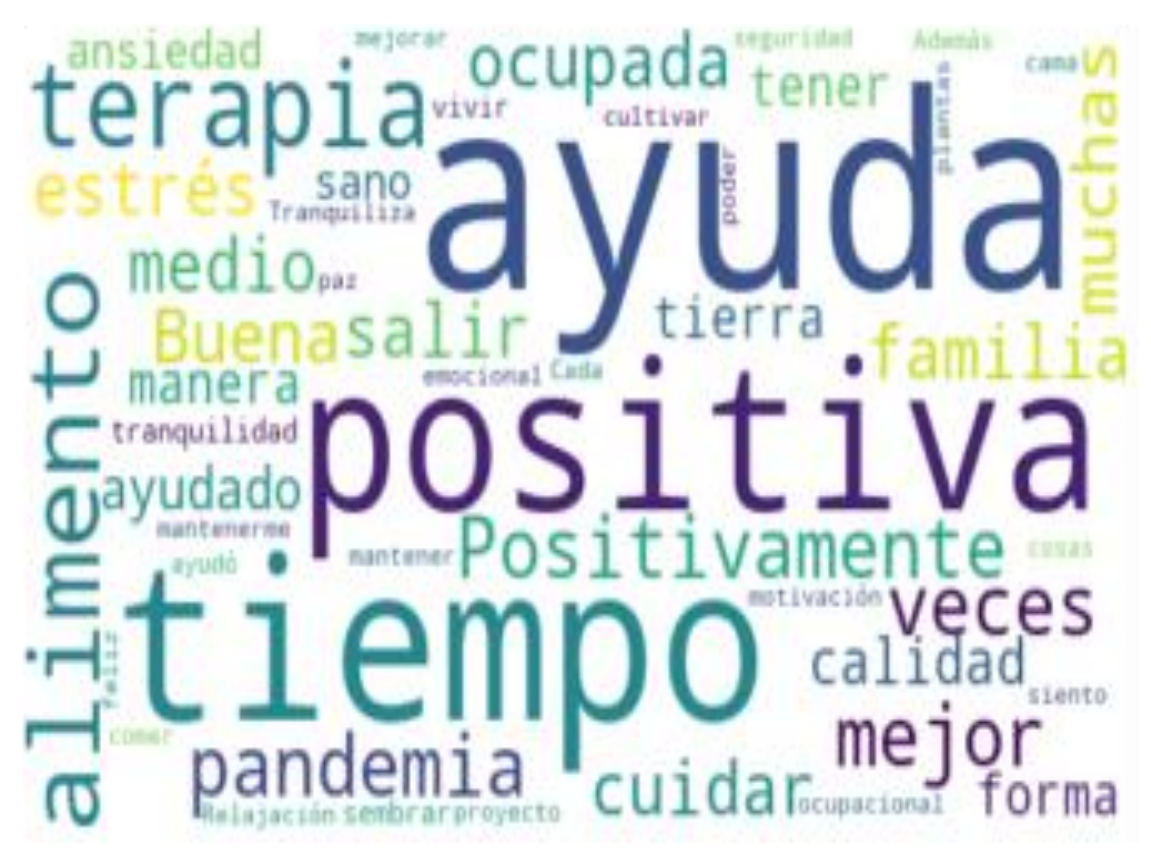

Fuente: Elaboración propia mediante el uso de Colaboratory.

\section{Ventajas y desventajas metodológicas}

Una de las grandes ventajas de emplear las aplicaciones de Google es que la información vertida en los formularios se vincula inmediatamente con lo programado en Colaboratory, lo que genera un análisis de tiempo real del fenómeno a estudiar, simplificando el lapso de procesamiento de la información. Y al estar anclado a la "nube", se prescinde de memoria RAM y de almacenamiento en el hardware. Aunado a que las personas que colaboran en la investigación pueden consultar los avances de forma remota.

Espacialmente, esta metodología permitió abarcar una escala nacional de la Agricultura Urbana, pues su aplicación ayudó a recolectar información en distintas entidades del país sin la necesidad de recorrerlo. Dando paso a un análisis más inmediato del fenómeno de interés.

Para distintas investigaciones, el financiamiento limita su óptimo desarrollo, por lo tanto, la utilización de estas herramientas brinda una ventaja sobre el aspecto económico, disminuyendo los costos de traslado, papelería, adquisición de software especializado para el análisis de datos (Atlas.ti., MAXQDA, NVIVO, etc.), personal y materiales. No obstante, se percibe que la falta de contacto personal puede, a los ojos del entrevistador o encuestador, perder elementos importantes como gestos, miradas e incluso posturas que ayuden a responder el "por qué" de la información obtenida, lo que podría ser una desventaja.

También, es importante considerar que no todos los individuos que practican la AU tienen acceso a esta tecnología (Internet), es decir, se podrá conocer la opinión y perspectiva de aquellos individuos que pueden adquirir este servicio, pero existirá un sesgo en la información 
recabada y también requiere de la posesión de un equipo (computadora, celular, tablet), y una serie de conocimientos para su manejo ${ }^{58}$. Además, una de las desventajas asociadas con las encuestas autoadministradas mediante Internet se relaciona con la dificultad para localizar muestras representativas ${ }^{59}$. Aunado a que esta encuesta está condicionada a la participación de las personas (disposición para contestar o no el formulario), sin mencionar que el creciente uso de encuestas online durante la pandemia ha provocado una saturación en diferentes plataformas que podría fomentar un estado de "hartazgo" por parte de la población objetivo, lo que generaría una desventaja asociada con la participación. En este sentido se propone generar redes de colaboración académica para evitar duplicar información, y se facilite la comunicación. No obstante, algunos estudios mencionan la rapidez del acceso de la población a la red lo que indica que estos sesgos podrían disminuir conforme pasa el tiempo ${ }^{60}$.

Es importante proponer o destacar metodologías que ayuden a la disminución de estas limitantes para poder abarcar un universo más grande de posibles respuestas. Una alternativa es la generación de información a partir de esta tecnología, para conocer las condiciones generales, actores clave y una línea base de información y que, posteriormente se apliquen metodologías sociales grupales mediante reuniones en línea, utilizando distintas plataformas (zoom, meet, teams, hangouts, bluejeans, telegram, whatsapp, etc) ${ }^{61}$. Esto supone, que para esta etapa ya se habrán identificado actores clave que auxilien en el proceso de organización y elección de un sitio en donde las personas puedan interactuar. De acuerdo con los resultados en la búsqueda de grupos en Facebook al menos tres estados presentaron una mayor participación y contemplando esto, es posible encontrar estrategias específicas de entrada al campo para profundizar en aquellas regiones con mayor participación, como el caso de grupos focales, entrevistas estructuradas o semiestructuradas.

Un estudio en Brasil sobre AU, en donde se aplicaron entrevistas, menciona que el proceso de recolección de información tardó dos meses (enero y febrero) ${ }^{62}$. Al comparar este tiempo con el de la aplicación de entrevistas que comprenden esta investigación, se puede decir que este fue un $68,3 \%$ menor. Y demuestra que esta herramienta ofrece una ventaja respecto a otras, como se ha mencionado en otros documentos que presumen la reducción del tiempo ${ }^{63}$.

Por último, existen casos de encuestas tradicionales que en poco tiempo obtienen gran cantidad de información, ejemplo de ello, fue un estudio colombiano que durante un periodo de 10 días obtuvo 385 encuestas, sin embargo, cabe aclarar que la encuesta se aplicó por interceptación en una zona comercial ${ }^{64}$, lo que implica un espacio reducido a diferencia de la propuesta actual que permitió conocer la opinión a escala nacional.

\footnotetext{
${ }^{58}$ Gibson, 2010 citado en Diaz de Rada, 2012, p. 199

<https://www.raco.cat/index.php/Papers/article/view/248512>.

${ }^{59}$ Díaz de Rada, 2012, p.198 <https://www.raco.cat/index.php/Papers/article/view/248512>; Dixon y Turner, 2006. DOI: 10.4018/978-1-59140-792-8.ch011.

60 Díaz de Rada, 2012, p.199 <https://www.raco.cat/index.php/Papers/article/view/248512>.

${ }^{61}$ Pacheco, $2020<$ https://www.iis.unam.mx/blog/etnografia-covid-19/>.

62 Comassetto et al., 2012 <https://www.scielo.br/pdf/rae/v53n4/v53n4a04.pdf>.

63 Dixon y Turner, 2006. DOI: 10.4018/978-1-59140-792-8.ch011.

64 Cancino, 2020 <http://www.revistaespacios.com/a20v41n07/20410709.html>.
} 


\section{Conclusiones}

La AU influye directamente en los aspectos económicos, sociales, ambientales y culturales; los aspectos económicos, resaltan su importancia en la disminución de coste de la vida respecto a la alimentación. En cuanto a los aspectos sociales, la Agricultura Urbana promueve varios beneficios, entre ellos la mejora de la salud física y psicológica o mental.

La influencia de la Agricultura Urbana en términos medioambientales se refleja mediante las estrategias de disminución de gases de efecto invernadero, reducción de residuos sólidos, conservación de semillas, conservación del suelo, y uso racional de los recursos. Mientras que el aspecto cultural es una parte inherente en la AU, que no solo es benéfica para mantener las tradiciones y permite a aquellas personas continuar con sus actividades independientemente del lugar en que se encuentren, sino que también preserva el conocimiento acerca del cuidado y producción de alimentos. Es importante el vínculo entre agricultura y salud, y esto se puede lograr a través de instituciones que promuevan políticas y programas para obtener un impacto en la población para llevar a cabo esta práctica.

Sin lugar a duda, la metodología aplicada a partir del uso de herramientas de gestión de la información a distancia brinda una excelente oportunidad para acercarse a la población objetivo mediante el uso de Internet, reduciendo costes y organizando la información obtenida. Las ventajas metodológicas encontradas, rondan los aspectos económicos, temporales y espaciales. Para el primer caso disminuye los costes en la implementación de los proyectos, mientras que, para el caso temporal, los tiempos de aplicación y obtención de respuestas disminuye, mientras que espacialmente permite abarcar una mayor escala.

Aplicar este tipo de metodologías, permite agilizar la obtención de datos. La creciente utilización de Python en las ciencias sociales puede conducir a una mejor aplicabilidad de los resultados en la investigación social ${ }^{65}$, para generar estrategias de acercamiento a través de distintas plataformas o elección de sitios de interés para acercarse al campo. Sin embargo, al definir la población objetivo, el acceso a Internet podría significar un sesgo en la información, lo que supone una desventaja a la que se añade la necesidad de tener equipos que permitan el acceso a este servicio y los conocimientos básicos de su manejo. Si bien existen ventajas para la obtención de información online, estos métodos podrían estar enfrentándose a un hartazgo social, por lo tanto, la creación de redes de colaboración para compartir información podría ser una opción viable para disminuir esta limitante.

Finalmente, el aporte del artículo consistió en proponer una metodología que ayude a conjuntar diversas aplicaciones de Google para la recolección de información de forma remota, el análisis de textos mediante su automatización y sin la necesidad de instalar un software. En las ciencias sociales, el uso de herramientas tecnológicas para el análisis del comportamiento humano ayuda a simplificar y sistematizar la información recabada. Esta propuesta fortalece las estrategias de investigación social emergentes en época de pandemia, proponiendo alternativas para disminuir las posibles limitantes en estos estudios. Y, en el contexto mexicano, nos permite entender una generalidad para aterrizar en aspectos más puntuales de temas y métodos de recogida de información. No obstante, aunado a esta aproximación metodológica se le deben

${ }^{65}$ Heiberger y Riebling, 2016, p. 9 <DOI: 10.1177/2059799115622763>. 
incorporar otras estrategias para facilitar y enriquecer la recolección de datos, con el objetivo de impulsar las investigaciones multimétodo.

\section{Bibliografía}

ABUNDIS, Víctor. Beneficios de las encuestas electrónicas como apoyo para la investigación. Tlatemoani: revista académica de investigación. [En línea]. Málaga: Universidad de Málaga, 19 de agosto de 2016, vol. 7, no 22, p. 168-186. $<$ https://dialnet.unirioja.es/servlet/articulo?codigo=7286080>.

AGROMÁTICA. Guía de huerto y jardinería. [En línea]. <https://www.agromatica.es/jardin/> [Consulta: 18 de enero de 2021].

AURORA UNIVERSITY. History of Urban Agriculture. [En línea]. $<$ https://online.aurora.edu/history-of-urban-agriculture/> [Consulta: 18 de febrero de 2021].

ARI, Niyazi y USTAZHANOV, Makhamadsulton. Matplotlib in python. In: 2014 11th International Conference on Electronics, Computer and Computation (ICECCO). IEEE. [En línea]. 2014. p. 1-6. 〈https://ieeexplore.ieee.org/document/6997585>.

BIRD, Steven; KLEIN, Ewan; LOPER, Edward. Natural language processing with Python Sebastopol: O'Reilly Media, Inc., 2009. 504 p.

BISONG, Ekaba. Building Machine Learning and Deep Learning Models on Google Cloud Platform. Ottawa: Apress, 2019. 709 p.

BOCCARDO, Giorgio y RUIZ, Felipe. RStudio para Estadística Descriptiva en Ciencias Sociales. [En línea]. $<$ https://www.researchgate.net/profile/Felipe_Ruiz15/publication/334308025_RStudio_para_ Estadistica_Descriptiva_en_Ciencias_Sociales_Manual_de_apoyo_docente_para_la_asignatu ra_Estadistica_Descriptiva_Carrera_de_Sociologia_Universidad_de_Chile_segunda_edicion/ links/5d23aafa92851cf4407274cf/RStudio-para-Estadistica-Descriptiva-en-Ciencias-

Sociales-Manual-de-apoyo-docente-para-la-asignatura-Estadistica-Descriptiva-Carrera-deSociologia-Universidad-de-Chile-segunda-edicion.pdf> [Consulta: 16 de febrero de 2021].

CANCINO, Yezid, et al. Comparación del comportamiento del consumidor en tiendas de descuento duro y grandes superficies. Revista Espacios. [En línea]. Caracas: Editorial espacios, 5 de marzo de 2020, vol. 41, no 07. <http://www.revistaespacios.com/a20v41n07/20410709.html>.

CANTOR, Kelly-M. Agricultura urbana: elementos valorativos sobre su sostenibilidad. Cuad. Desarro. Rural. [En línea]. Bogotá: Pontificia Universidad Javeriana, 30 de diciembre de 2010, vol.7, n.65. p.59-84. <http://www.scielo.org.co/scielo.php?script=sci_arttext\&pid=S0122$14502010000200004 \& \operatorname{lng}=\mathrm{es} \& \mathrm{nrm}=\mathrm{iso}>$.

CHÁVEZ, Adela y PALACIOS, Juan. Importancia de los cursos de agricultura urbana ante el cambio climático y la seguridad alimentaria en México. Revista DELOS Desarrollo Local 
Sostenible. [En línea]. España: Universidad de Málaga 2017, vol.10, n.28, p.1-13. $<$ https://dialnet.unirioja.es/servlet/articulo?codigo=6500073>.

COMASSETTO, Bruno Henrique, SOLALINDE, Gabriela Z.P., DE SOUZA João Vicente Rosa, TREVISAN, Marelo, ZILIO Abadala, Paulo Ricardo y VARGAS Rossi, Carlos Alberto. Nostalgia, anticonsumo simbólico y bienestar: la agricultura urbana. Revista de Administração de Empresas. [En línea]. São Paulo: Escola de Administração de Empresas de São Paulo da Fundação Getulio Vargas, 2012, vol. 53, no. 4, p. 364-375. <https://www.scielo.br/pdf/rae/v53n4/v53n4a04.pdf >.

DEPAOLO, Concetta y WILKINSON, Kelly. Get your head into the clouds: Using word clouds for analyzing qualitative assessment data. TechTrends. [En línea]. Suiza: Springer, 21 de abril de 2014, vol. 58, no 3, p. 38-44. <https://link.springer.com/article/10.1007/s11528014-0750-9>.

DEGENHART, Barbara. La agricultura urbana: un fenómeno global. Nueva sociedad. [En línea]. Buenos Aires: Nueva Sociedad, 2016, $\mathrm{n}^{\mathrm{o}}$ 262, p. 133-146. $<$ https://www.nuso.org/articulo/la-agricultura-urbana-un-fenomeno-global/>.

DÍAZ DE RADA, Vidal. Ventajas e inconvenientes de la encuesta por Internet. Papers: revista de sociologia. [En línea]. Barcelona: Universidad Autónoma de Barcelona, enero de 2012, vol.97 nº1, p. 193-223 <https://www.raco.cat/index.php/Papers/article/view/248512>.

DIXON, Ronda; TURNER Rodney. Electronic vs. Conventional Surveys. In REYNOLDS, Rodney A.; WOODS, Robert; BAKER, Jason D. (ed.). Handbook of research on electronic surveys and measurements. London: Idea Group Reference, 2006. p. 104-111. DOI: 10.4018/978-1-59140-792-8.ch011

FACEBOOK. Resultados de búsqueda. [En línea]. <https://www.facebook.com/> [Consulta: 10 de enero de 2021].

FAO (Organización de las Naciones Unidas para la Agricultura y la Alimentación). La agricultura urbana y su contribución a la seguridad alimentaria. Sistematización del Proyecto Piloto AUP en Honduras. Honduras: FAO, 2012. 32 p. $<$ http://www.fao.org/3/as174s/as174s.pdf >.

FAO. Cultivos para la alimentación diaria. Mejorando la nutrición a través de huertos y granjas familiares. Manual de capacitación para trabajadores de campo en América latina y el Caribe. [En línea] <http://www.fao.org/3/V5290S/v5290s28.htm> [Consulta: 05 de enero de 2021].

FAO y CEPAL. Sistemas alimentarios y COVID-19 en América Latina y el Caribe: La oportunidad de la transformación digital. [En línea]. Santiago: FAO, 10 de junio de 2020, Boletín $n^{\circ} 8$. <https://repositorio.cepal.org/bitstream/handle/11362/45722/1/ca9508_es.pdf>.

GOBIERNO DE MÉXICO. Jornada Nacional de Sana Distancia. [En línea]. <https://www.gob.mx/cms/uploads/attachment/file/541687/Jornada_Nacional_de_Sana_Dista ncia.pdf> [Consulta: 28 de diciembre de 2020]. 
GONZÁLEZ, Felipe. Big data, algoritmos y política: las ciencias sociales en la era de las redes digitales. Cinta de Moebio. Revista de Epistemología de Ciencias Sociales. [En línea]. Santiago de Chile: Universidad de Chile, 19 de agosto de 2019, $\mathrm{n}^{\circ}$ 65, p. 267-280. $<$ https://cintademoebio.uchile.cl/index.php/CDM/article/view/54210>.

GOOGLE, Productos. [En línea]. <https://about.google/intl/es-419/products/> [Consulta: 8 de febrero de 2021].

GORDILLO G., MÉNDEZ J. O. Seguridad y soberanía alimentarias. Organización de las Naciones Unidas para la Agricultura y la Alimentación. [En línea] Chile. <file:///E:/Art\%C3\%ADculo\%20forest/ax736s.pdf>.

GRAJALES G.Angie Tatiana., PEÑALOZA G. Yessica Alejandra. Aspectos de la salud mental involucrados en la construcción de huertas. Universidad Cooperativa de Colombia. [En línea]. $\quad$ Colombia. 2019. $<$ https://repository.ucc.edu.co/bitstream/20.500.12494/13421/1/2019_aspectos_salud_mental. pdf $>$

HAWKES, Corinna y RUEL, Marie. The links between agriculture and health: an intersectoral opportunity to improve the health and livelihoods of the poor. Bulletin of the World Health organization. [En línea]. Ginebra: World Health Organization, diciembre de 2006, vol. 84, $\mathrm{n}^{\circ}$ 12, p. 984-990. <https://www.scielosp.org/pdf/bwho/2006.v84n12/984-990/en>.

HEIBERGER, Raphael H.; RIEBLING, Jan R. Installing computational social science: Facing the challenges of new information and communication technologies in social science. Methodological Innovations. [En línea]. Reino Unido: SAGE Publications, 2 de marzo de 2016, vol. 9, p.1-11. DOI: 10.1177/2059799115622763

IFT (Instituto Federal de Telecomunicaciones). Uso de las TIC y actividades por internet en México: Impacto de las características Sociodemográficas de la población (Versión 2019). [En línea]. México. IFT 16 de enero de 2020 p. 1-94 <http://www.ift.org.mx/estadisticas/uso-delas-tic-y-actividades-por-internet-en-mexico-impacto-de-las-caracteristicassociodemograficas $-0>$.

JOSIANE Kennard Nicole y HUGH Bamford Robert. Urban Agriculture: Opportunities and Challenges for Sustainable Development. inbook. [En línea]. Suiza: Springer, Cham, 03 de diciembre de 2019 <https://doi.org/10.1007/978-3-319-69626-3_102-1>.

KOZAI, Toyoki; NIU Genhua y TAKAGAKI Michiko. PFAL Business and R\&D in the World: Current Status and Perspectives. Plant Factory. Chapter 3 de Plant Factory an Indoor Vertical Farming System for Efficient Quality Food Production. [En línea]. Estados Unidos: Academic Press. 2016, p. 35-68. <https://doi.org/10.1016/B978-0-12-801775-3.00003-2>.

LOVELL, Sarah. Multifunctional Urban Agriculture for Sustainable Land Use Planning in the United States. Sustainability. [En línea]. Basel: MDPI, 4 de agosto de 2010. Vol. 2, no. 8, p. 2499-2522 <https://www.mdpi.com/2071-1050/2/8/2499>. 
MARCOS, Sara. Nuevas Tendencias Alimentarias: Vegetarianismo y Ortorexia Nerviosa. Tesis (Grado en Enfermería) Director: Isabel Calvo Viñuela. Madrid: Universidad Autónoma de Madrid, 2019. <https://repositorio.uam.es/bitstream/handle/10486/687899/marcos_retuerta_saratfg.pdf?sequ ence $=1 \&$ isAllowed $=\mathrm{y}>$.

MEJÍAS M. A. I. Contribución de los huertos urbanos a la salud. Hábitat y Sociedad [En línea]. $\begin{array}{lllllll}\text { España, } 21 \text { de enero de 2014. } & \mathrm{N}^{\circ} & 6, & \text { p. } & 85-103\end{array}$ <https://institucional.us.es/revistas/habitat/6/HyS\%20n06a04.pdf>.

McCLINTOCK, Nathan. Why farm the city? Theorizing urban agriculture through a lens of metabolic rift. Cambridge Journal of Regions, Economy and Society. [En línea]. Oxford: Oxford University, 25 de marzo de 2010 vol. 3, no 2, p. 191-207. DOI:10.1093/cjres/rsq005

MENDEZ Marlon; RAMÍREZ Luz; ALZATE Alejandra. La práctica de la agricultura urbana como expresión de emergencia de nuevas ruralidades: reflexiones en torno a la evidencia empírica. Cuadernos de Desarrollo Rural, [En línea]. Colombia: Pontificia Universidad Javeriana, 2005, no 55, p. 51-70. <https://www.redalyc.org/articulo.oa?id=11705504>.

NADAL, Ana, et al. Social perception of urban agriculture in Latin-America. A case study in Mexican social housing. Land Use Policy. [En línea]. Holanda: Elsevier, 14 de marzo de 2018, vol. 76 , p. 719-734. <https://www.sciencedirect.com/science/article/abs/pii/S0264837717301096>.

OLIDEN, Paula Elosua. ¿Existe vida más allá del SPSS? Descubre R. Psicothema. [En línea]. Asturias: Colegio oficial de psicólogos del principado de Asturias, 2009, vol. 21, nº 4, p. 652$655<\mathrm{http}: / / \mathrm{www} . \mathrm{psicothema.com/pdf/3686.pdf>}$.

OMS (Organización Mundial de la Salud). Cronología de la respuesta de la OMS a la COVID19. [En línea] <https://www.who.int/es/news/item/29-06-2020-covidtimeline> [Consulta: 17 de enero de 2021].

ONU (Organización Mundial de las Naciones Unidas). Agricultura urbana, una opción ante la emergencia sanitaria. [En línea] <https://coronavirus.onu.org.mx/agricultura-urbana-unaopcion-ante-la-emergencia-sanitaria> [Consulta: 17 de enero de 2021].

ORSINI Francesco, KAHANE Remi, NONO-WOMDIM Remi y GIANQUINTO Giorgio. Urban agriculture in the developing world: a review. Agron. Sustain. Dev. [En línea]. Suiza: Springer, 09 de mayo de 2013, vol. 33, s.pág. DOI: 10.1007/s13593-013-0143-Z

PACHECO Raúl. Etnografías en tiempos de COVID-19. [En línea]. Entrevista realizada por Laura Montes de Oca, 28 de agosto de $2020<$ https://www.iis.unam.mx/blog/etnografiacovid-19/> [Consulta: 20 de febrero de 2021].

PORTAL FRUTÍCOLA. Guía para principiantes para el cultivo de hortalizas y frutas en espacios reducidos. [En línea] <https://www.portalfruticola.com/noticias/2018/11/12/guiapara-principiantes-para-el-cultivo-de-hortalizas-y-frutas-en-espacios-reducidos/>. 
PEÑA SARMIENTO N.P., VESELOVSCHI Paula. La Agricultura Urbana como herramienta para el bienestar de las personas mayores del Centro de Protección Social Bosque Popular de Bogotá. Universidad Externado de Colombia. Colombia. Junio 2019 [En línea]. $<$ https://bdigital.uexternado.edu.co/bitstream/001/2247/1/DQA-spa-2019-

La_agricultura_urbana_como_herramienta_para_el_bienestar_de_las_personas_mayores $>$.

PHILPOTT Stacy M.; EGERER Monika H.; BICHIER Peter; COHEN Hamutahl; COHEN Roseann; LIERE Heidi; JHA Shalene; LIN Brenda. Gardener demographics, experience, and motivations drive differences in plant species richness and composition in urban gardens. Ecology and Society. USA. 2020, $\mathrm{n}^{\circ} 25$ vol 4:8 <https://doi.org/10.5751/ES-11666-250408>.

ROVELO, Renata. Huertos urbanos, solución a la vulnerabilidad alimentaria por COVID. $L a$ mirada de la Academía, Instituto de Investigación Aplicada y Tecnología (InIAT) IBERO Ciudad de México. [En línea]. <https://ibero.mx/prensa/huertos-urbanos-solucion-lavulnerabilidad-alimentaria-por-covid $>$.

SRINIVASA, K. G.; SIDDESH, G. M.; SRINIDHI, H. Network data analytics: a hands-on approach for application development. Switzerland: Springer, 2018. 398 p.

TALENS, Raúl. La relación entre los casos de Covid-19 y su impacto en Twitter. Tesis Grado en Ingeniería Informática. Directores: César Ferri Ramírez y Fernando Martínez Plumed. Valencia: Universitat Politècnia de 2020 <https://riunet.upv.es/handle/10251/156675>.

VANDERPLAS, Jake. Python data science handbook: Essential tools for working with data. USA: O'Reilly Media, Inc., 2016. 530 p.

VÁSQUEZ MORENO, Larissa, 2010. La agricultura urbana como elemento promotor de la sustentabilidad urbana. situación actual y potencial en San Cristóbal de las casas, Chiapas. Tesis de maestría. El Colegio de la Frontera Norte. Tijuana México. [En línea]. <https://www.colef.mx/posgrado/wp-content/uploads/2011/04/TESIS-V\%C3\%A1squezMoreno-Larissa-MAIA.pdf $>$.

WIEMKEN, Timothy, et al. Googling your hand hygiene data: Using Google Forms, Google Sheets, and $\mathrm{R}$ to collect and automate analysis of hand hygiene compliance monitoring. American journal of infection control. [En línea]. Amsterdam: Elsevier, junio de 2018, vol 46 $\begin{array}{llll}\mathrm{n}^{\mathrm{o}} & 6, & \mathrm{p} . & 617-619\end{array}$ <https://www.sciencedirect.com/science/article/abs/pii/S0196655318300312>.

WILSON, Robert E.; GOSLING, Samuel D.; GRAHAM, Lindsay T. A review of Facebook research in the social sciences. Perspectives on psychological science. [En línea]. Reino Unido: SAGE Publications, 16 de mayo de 2012, vol. 7, $\mathrm{n}^{\mathrm{o}} 3$, p. 203-220. <https://journals.sagepub.com/doi/10.1177/1745691612442904>.

ZAAR, Miriam Hermi. A agricultura Urbana e Periurbana (AUP) no marco da soberania alimentar. Sociedade e Território, vol. 27, $\mathrm{n}^{\mathrm{o}} 3$, p. 26-44, <https://periodicos.ufrn.br/sociedadeeterritorio/article/view/7870> [ISSN 21778396]. 
Contexto general de la Agricultura Urbana en México durante la pandemia...

ZAAR, Miriam Hermi. Agricultura urbana: algunas reflexiones sobre su origen y expansión. Biblio 3W. Revista Bibliográfica de Geografía y Ciencias Sociales. [En línea]. Barcelona: Universidad de Barcelona, 15 de octubre de 2011, Vol. XVI, n ${ }^{\circ} 944$. <http://www.ub.es/geocrit/b3w-944.htm> [ISSN 1138-9796].

\section{Apéndice I.}

Encuesta aplicada en la presente investigación, misma que fue enviada a los grupos de Facebook previamente descritos en la sección de metodología.

\section{Agricultura urbana en el contexto COVID}

Objetivo: Conocer el panorama general de la agricultura urbana en México y su relevancia ante el contexto COVID.

Nota: La información será usada para fines académicos; los nombres de las personas no serán solicitados para mantener su anonimato.

Datos generales.

Estado: Municipio: Localidad:

Edad:

Sexo: Hombre Mujer Prefiero no decirlo

\section{Preguntas.} Mujer - Prefiero no decirlo

1. ¿Qué te motivó a practicar agricultura?

2. ¿Qué ventajas trae consigo la agricultura urbana?

3. Describe con una palabra o frase, tu relación con la actividad agrícola.

4. ¿Cómo aprendiste a sembrar?

5. ¿Cuántas horas a la semana inviertes en el cuidado de tu huerto?

6. ¿Disminuirá el tiempo que le dedicas al huerto con el fin de la pandemia?

- Si _ No - Tal vez

7. ¿Qué has sembrado? (puedes seleccionar varias opciones)

_ Acelgas

- Apio

_ Aromáticas (romero, albahaca, menta, hierbabuena, etc.)

- Betabel

— Berries (frambuezas, fresas, moras, arándanos, etc.)

— Brócoli

_ Calabaza

_ Cebolla

_ Chiles

_ Cilantro 
- Col

_ Coliflor

_ Espinacas

_ Jitomates

— Lechuga

— Leguminosas (chícharo, haba, frijol, etc.)

- Maíz

— Melón

_ Papa

_ Rábano

- Sandía

Otra Añadir opción

8. ¿Dónde adquiriste tus semillas?

9. ¿Cuál es el tamaño promedio de tu huerto?

Menos de 1 metro cuadrado

— Entre 1 y 2 metros cuadrados

_ Entre 3 y 5 metros cuadrados

_ Entre 6 y 8 metros cuadrados

— Entre 9 y 11 metros cuadrados

_ Más de 11 metros cuadrados

10. ¿Cómo te ha influido la agricultura urbana durante la pandemia?

¡MUCHAS GRACIAS!

Ficha bibliográfica:

MIRANDA GÓMEZ, Omar; AGUILAR-GÓMEZ, Carlos Rubén. Contexto general de la Agricultura Urbana en México durante la pandemia: uso de aplicaciones de Google para el análisis social. Ar@cne. Revista Electrónica de Recursos de Internet sobre Geografia y Ciencias Sociales. Barcelona: Universidad de Barcelona, I de septiembre de 202I, vol. XXV, $n^{\circ} 258$. DOI: https://doi.org/I0.1344/ara2021.258.34042 